\title{
Erratum to: The Role of Sleeve Gastrectomy in Reducing Cardiovascular Risk
}

\author{
Luciana Tromba ${ }^{1}$ - Francesco Tartaglia ${ }^{1} \cdot$ Sabino Carbotta $^{1} \cdot$ Nadia Sforza $^{1}$ • \\ Fabio Pelle ${ }^{1}$ - Vanessa Colagiovanni ${ }^{2} \cdot$ Giovanni Carbotta $^{3} \cdot$ Stefania Cavaiola $^{4}$. \\ Giovanni Casella ${ }^{1}$
}

Published online: 14 November 2016

(C) Springer Science+Business Media New York 2016

Erratum to: OBES SURG

DOI 10.1007/s11695-016-2441-4

In the original article the names of all the authors were presented incorrectly. They are correct here.

The online version of the original article can be found at http://dx.doi. org/10.1007/s11695-016-2441-4.

Francesco Tartaglia
francesco.tartaglia@uniroma1.it
Luciana Tromba
luciana.tromba@uniroma1.it
Sabino Carbotta
sabino.carbotta@uniroma1.it
Nadia Sforza
nadia@ hotmail.it
Fabio Pelle
fabiopelle75@gmail.com
Vanessa Colagiovanni
vanessacolagiovanni@hotmail.com

Giovanni Carbotta giovanni.c@tiscali.it

Stefania Cavaiola cavaiola@tiscali.it

Giovanni Casella giovanni.casella@uniroma1.it

1 Surgical Sciences Department, "Sapienza" University of Rome, Rome, Italy

2 Gynecological, Obstetric Sciences Department and Urological Sciences, "Sapienza" University of Rome, Rome, Italy

3 Department of Experimental Medicine, "Sapienza" University of Rome, Rome, Italy

4 CNR, IBB, Rome, Italy 